Atomic and molecular hydrogen gas temperatures in a low-pressure helicon plasma

This content has been downloaded from IOPscience. Please scroll down to see the full text.

2015 Plasma Sources Sci. Technol. 24045003

(http://iopscience.iop.org/0963-0252/24/4/045003)

View the table of contents for this issue, or go to the journal homepage for more

Download details:

IP Address: 130.56.94.131

This content was downloaded on 23/06/2015 at 02:52

Please note that terms and conditions apply. 


\title{
Atomic and molecular hydrogen gas temperatures in a low-pressure helicon plasma
}

\author{
Cameron M Samuell and Cormac S Corr \\ Plasma Research Laboratory, Research School of Physics and Engineering, Australian National \\ University, Canberra, Australia \\ E-mail: cameron.samuell@anu.edu.au and cormac.corr@anu.edu.au
}

Received 8 February 2015, revised 6 May 2015

Accepted for publication 18 May 2015

Published 19 June 2015

\begin{abstract}
Neutral gas temperatures in hydrogen plasmas are important for experimental and modelling efforts in fusion technology, plasma processing, and surface modification applications. To provide values relevant to these application areas, neutral gas temperatures were measured in a low pressure ( $<10$ mTorr) radiofrequency helicon discharge using spectroscopic techniques. The atomic and molecular species were not found to be in thermal equilibrium with the atomic temperature being mostly larger then the molecular temperature. In low power operation $(<1 \mathrm{~kW})$, the molecular hydrogen temperature was observed to be linearly proportional to the pressure while the atomic hydrogen temperature was inversely proportional. Both temperatures were observed to rise linearly with input power. For high power operation (5-20 kW), the molecular temperature was found to rise with both power and pressure up to a maximum of approximately $1200 \mathrm{~K}$. Spatially resolved measurements near a graphite target demonstrated localised cooling near the sample surface. The temporal evolution of the molecular gas temperature during a high power $1.1 \mathrm{~ms}$ plasma pulse was also investigated and found to vary considerably as a function of pressure.
\end{abstract}

Keywords: plasma, RF, helicon, hydrogen, gas temperature, neutral temperature

(Some figures may appear in colour only in the online journal)

\section{Introduction}

The temperature of neutral gas species in a plasma can be important both for basic plasma processes and for material modification. Gas temperature impacts strongly on the gasphase chemistry where it can be a limiting factor in chemical processes and can influence particle densities, spatial distributions, and transport [1,2]. Gas temperature can also contribute to the heating of reactor walls and sample surfaces causing blistering [3], bubbling, and nanostructure formation [4].

The gas temperature is also important for numerical modelling and simulations. It has been stated that 'it is meaningless to attempt plasma modelling without knowing the kinetic temperature of the neutrals' [2]. Using hydrogen as an example, gas temperature can affect the atomic hydrogen density both directly, through heavy particle reaction rates, and indirectly, for example through changes in the atomic loss coefficient [5]. However, the gas temperature is often assumed to be equal to the room temperature as is the case in the classical description of low-pressure plasma systems [6] or constant with respect to operating parameters $[5,7]$.

Hydrogen plasmas are of particular interest for applications in plasma processing, fusion energy, negative ion sources, and neutral beam injection systems. In the case of hydrogen, the measurement and influence of gas temperature is complicated by the presence of both atomic and molecular neutral species that may not be in thermal equilibrium. However, there have only been a very limited number of measurements for hydrogen temperatures in low pressure systems. Measurements in hydrogen plasmas tend to focus on microwave discharges [2,8-12] or, in the case of radio frequency ICP or helicon sources, high pressure regimes $[1,6,13,14]$ 
with the only exception being the recent work conducted by Galante et al on a helicon source at low pressure (1-50 mTorr) and with powers $<5 \mathrm{~kW}$ [15].

In this paper, atomic and molecular hydrogen neutral gas temperatures are presented. Experiments were conducted in the MAGPIE system at powers up to $20 \mathrm{~kW}$ and pressures in the range of 3-10 mTorr. To the author's knowledge, the temperatures presented here represent the most detailed characterisation of neutral temperatures in a low pressure ( $<20$ mTorr) RF discharge to date and the first measurements in a high-power helicon system.

\section{Experimental setup}

The MAGPIE reactor is a low pressure magnetically enhanced helicon reactor based at the Australian National University. Detailed descriptions of the MAGPIE experimental system have been given previously and so only a brief overview is presented here [16-18].

MAGPIE's vacuum chamber consists of a borosilicate source tube connected to a stainless steel target chamber. For material studies, a target holder was inserted into the target chamber that placed a sample approximately $15 \mathrm{~cm}$ downstream of the source region.

Power delivery was achieved using two separate RF supply systems defined here as 'low-power' and 'high-power' setups. The low-power system operates at $13.56 \mathrm{MHz}$ and consisted of a Rohde and Schwarz SM300 signal generator driving an ENI A-150 $150 \mathrm{~W}$ pre-amplifier that, in turn, supplied an Alpha 77Dx generator. The Alpha power supply delivers up to $5 \mathrm{~kW}$ of pulsed power and up to $1.5 \mathrm{~kW}$ steady-state power for hydrogen operation. The high-power setup comprised an Agilent 3350A signal generator, HLA 300 plus HF linear amplifier, and a RM KL-400 linear amplifier connected to a $20 \mathrm{~kW}$ AWA HF pulse amplifier. This system could supply between 5 and $20 \mathrm{~kW}$ at $7 \mathrm{MHz}$. It was optimised to supply pulses of up to $8000 \mathrm{RF}$ cycles at around $10 \mathrm{~Hz}$ which resulted in $1.14 \mathrm{~ms}$ pulse length and a $1 \%$ duty cycle. In both cases power was delivered through a $\pi$-type matchbox to a lefthanded $22 \mathrm{~cm}$ Nagoya III antenna with a $180^{\circ}$ twist.

A DC magnetic field was applied using two sets of five water cooled solenoids surrounding the source region and target chamber. The source and target solenoids were supplied with currents of $50 \mathrm{~A}$ and $100 \mathrm{~A}$ respectively to produce a relatively weak magnetic field of around $50 \mathrm{G}$ at the sample holder.

MAGPIE typically exhibits hydrogen plasma electron temperatures in the $1-10 \mathrm{eV}$ range and electron densities up to $n_{\mathrm{e}}=10^{19} \mathrm{~m}^{-3}$. These basic characteristics provide an opportunity to measure neutral temperatures in a regime relevant to plasma processing, the divertor region of a fusion reactor, plasma-material interaction experiments and low-pressure plasmas in general.

\section{Diagnostic methods}

Atomic and molecular hydrogen neutral gas temperatures were measured using two distinct diagnostic methods. The atomic temperature was measured using the Doppler broadening of Balmer- $\alpha$ emission. For molecular hydrogen temperatures, the relative intensities of the Q-branch of Fulcher- $\alpha$ ro-vibrational transitions were used. By using two entirely separate techniques, the measurements could be made simultaneously and compared. While both diagnostic techniques are relatively straightforward, care must be taken in each case to ensure the technique's validity for a given plasma system and operating regime.

\subsection{Doppler broadening}

The width of observed plasma emission lines is related to species temperature through Doppler broadening. The random motion of atoms in the plasma owing to their kinetic energy (temperature) causes a broadening of the emission that is given by [8]:

$$
\Delta \lambda_{D}=\lambda_{c} \sqrt{\frac{8 \ln (2) T k_{\mathrm{B}}}{m c^{2}}}
$$

where $\lambda_{c}$ is the centre wavelength, $T$ is the temperature of the emitting species, $m$ is the mass of the emitting species, $k_{\mathrm{B}}$ is the Boltzmann constant, and $c$ is the speed of light. The small mass of hydrogen atoms makes it ideal for Doppler measurements as the effect is greater for small $m$.

Aside from Doppler broadening, there are several other broadening mechanisms that can contribute to the broadening of plasma emission. These mechanisms include: the natural line width of the transition; stark broadening produced as a result of electric fields in the plasma; Zeeman broadening due to the static magnetic field; the optical thickness of the plasma; and instrument broadening caused by the detection optics. The magnitude of each of these effects must be calculated for accurate calculation of the Doppler width. For reference, the magnitude of the Doppler broadening expected for a single hydrogen spectral line at a wavelength around $656 \mathrm{~nm}$ and emitting at a temperature of $600 \mathrm{~K}$ is on the order of $1 \times 10^{-2} \mathrm{~nm}$, calculated using equation (1).

The smallest contribution to the total broadening comes from the natural line broadening which has a maximum contribution of $10^{-5} \mathrm{~nm}$ and so is negligible compared to even a very modest Doppler width [19].

The magnitude of broadening by Zeeman splitting can be approximated as $\Delta \lambda_{Z} \sim 10^{-9} \lambda^{2}|B|$ in Angstroms where $|B|$ is in $\mathrm{kG}$. The magnetic field in the vicinity of the measurement position is approximately $50 \mathrm{G}$. Using this field strength, the magnitude of Zeeman splitting broadening is approximately $2 \times 10^{-5} \mathrm{~nm}$ and so can be ignored.

The FWHM of Stark-broadened Balmer $\alpha$ emission, $\Delta \lambda_{\mathrm{S}}$, can be calculated assuming ion collisions play a dominant role [20]. In this case,

$$
\Delta \lambda_{\mathrm{S}}=1.55 \times 10^{-4} \frac{n_{\mathrm{e}} \lambda_{0}^{2}}{\pi c} \sqrt{\frac{m_{\mathrm{i}}}{2 m_{\mathrm{e}} T_{\mathrm{i}}}}
$$

$$
\times\left[27.54+\ln \left(\frac{2 T_{d} T_{\mathrm{i}} m_{\mathrm{e}}}{n_{\mathrm{e}} m_{\mathrm{i}}}\right)\right]
$$




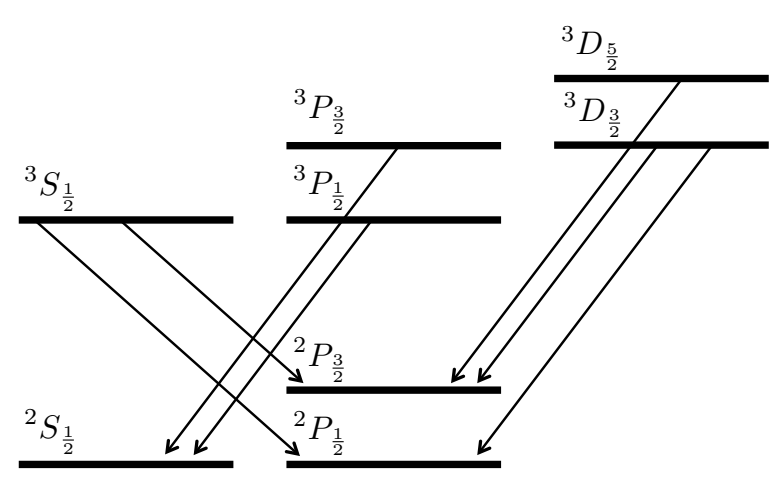

Figure 1. Energetic diagram of the fine structure transitions associated with the $n=3 \rightarrow n=2$ Balmer- $\alpha$ emission.

where $T_{\mathrm{e}}$ and $T_{\mathrm{i}}$ are the electron and ion temperatures in units of Kelvin, $n_{\mathrm{e}}$ is the electron density in $\mathrm{cm}^{-3}, m_{\mathrm{e}}$ and $m_{\mathrm{i}}$ are the electron and ion masses, $c$ is the speed of light, $\lambda_{o}$ is the centre wavelength, and $T_{D} \equiv T_{\mathrm{e}} T_{\mathrm{i}} /\left(T_{\mathrm{e}}+T_{\mathrm{i}}\right)$ [20]. To make an estimation of Stark broadening, $n_{\mathrm{e}}=10^{19} \mathrm{~m}^{-3}$ is the upper limit of electron densities observed in MAGPIE [16], ion temperatures were approximated to reach a maximum of about 5800 $\mathrm{K}$ at low pressures (1 mTorr) [21], electron temperatures were observed up to approximately $5 \mathrm{eV}$ in MAGPIE [16], and the heaviest ion expected in low pressure hydrogen plasmas was $\mathrm{H}_{3}^{+}$. With these assumptions, an upper-bound for Stark broadening was calculated to be approximately $3 \times 10^{-3} \mathrm{~nm}$ and so was neglected. It was not expected that there will be significant electric fields in the MAGPIE plasma that contribute to greater Stark broadening.

The optical thickness of the plasma may have an effect on the observed $\mathrm{H}_{\alpha}$ line broadening. By calculating the effective path length, $\tau$, it can be determined whether a given plasma is optically thick. For a given frequency, $\omega$, the effecive optical path length is given by $\tau_{\omega}(L)=\sigma_{\omega} n_{H} L$ for a physical path length $L$, absorption cross section, $\sigma_{\omega}$, and atomic hydrogen density, $n_{\mathrm{H}}$ [22]. To gain an estimation of $n_{\mathrm{H}}$, it is noted that Galante et al measured atomic hydrogen densities of approximately $5 \times 10^{19} \mathrm{~m}^{-3}$ in a helicon system operating under similar operating conditions to the present study [15]. Taking $L=0.1 \mathrm{~m}$ and $n_{H}=1 \times 10^{19} \mathrm{~m}^{-3}$ as an upper bound, $\tau_{\omega}=0.01$. Since $\tau_{\omega}<1$, the plasma is optically thin and self absorption of the Balmer- $\alpha$ line can be neglected as a source of broadening.

Therefore, the only broadening mechanisms expected to significantly contribute to an observed spectra are Doppler broadening and the apparatus broadening.

3.1.1. Fine structure splitting. The $\mathrm{H}_{\alpha}$ transition from the $n=3$ state to the $n=2$ state is composed of seven individual fine structure transitions. An energetic diagram of these transitions is shown in figure 1. Each of the seven fine structure transitions are broadened individually and therefore, given their spread of frequencies, contribute significantly to the total broadening observed in the $\mathrm{H}_{\alpha}$ line. At temperatures below approximately $1500 \mathrm{~K}$, this fine structure must be taken into account [23]. The experimental $\mathrm{H}_{\alpha}$ emission was considered as the sum of seven separate gaussians to separate the Doppler broadening effects from the broadening caused by the
Table 1. $\mathrm{H}_{\alpha}$ fine structure transition data used for calculating the individual transition contributions to $\mathrm{H}_{\alpha}$ broadening taken from the NIST online database for atomic data [24].

\begin{tabular}{lll}
\hline Transition & Wavelength [nm] & $\mathrm{S}_{\mathrm{i}, \mathrm{k}}$ \\
\hline${ }^{2} \mathrm{D}_{3 / 2} \rightarrow{ }^{2} \mathrm{P}_{1 / 2}$ & 656.2709702 & 30.089 \\
${ }^{3} \mathrm{P}_{3 / 2} \rightarrow{ }^{2} \mathrm{~S}_{1 / 2}$ & 656.2724827 & 12.537 \\
${ }^{3} \mathrm{~S}_{1 / 2} \rightarrow{ }^{2} \mathrm{P}_{1 / 2}$ & 656.2751807 & 0.58769 \\
${ }^{3} \mathrm{P}_{1 / 2} \rightarrow{ }^{2} \mathrm{~S}_{1 / 2}$ & 656.2771533 & 6.2688 \\
${ }^{3} \mathrm{D}_{5 / 2} \rightarrow{ }^{2} \mathrm{P}_{3 / 2}$ & 656.2851769 & 54.162 \\
${ }^{3} \mathrm{D}_{3 / 2} \rightarrow{ }^{2} \mathrm{P}_{3 / 2}$ & 656.2867336 & 6.0181 \\
${ }^{3} \mathrm{~S}_{1 / 2} \rightarrow{ }^{2} \mathrm{P}_{3 / 2}$ & 656.2909442 & 1.1756 \\
\hline
\end{tabular}

separation of fine structure states. Using data obtained from the NIST online atomic spectra database [24], the contributions of each of the fine-structure components were calculated, an example of which is shown in figure 2(a) for a 600 $\mathrm{K}$ emitting system. Wavelengths for each of the fine-structure transitions along with relevant atomic data used for calculating emission profiles is summarised in table 1. The effect of varying the temperature of an $\mathrm{H}$-atom system is shown in figure 2(b).

3.1.2. Diagnostic setup. $\mathrm{H}_{\alpha}$ emission was measured on the MAGPIE reactor using a $1 \mathrm{~m}$ Czerny-Turner spectrometer fitted with an Ames Photonic Inc. LARRY 2048 linear array CCD detector and a $2400 \mathrm{~g} \mathrm{~mm}^{-1}$ diffraction grating. An optical fibre was used to collect light remotely which was viewed by the spectrometer through an adjustable slit set to $50 \mu \mathrm{m}$. To make an estimation of the apparatus broadening of the optical system, the emission from a $656 \mathrm{~nm}$ diode laser was measured. The spectral broadening from a laser source is extremely small and provides a good indication of the optical system's broadening of the signal. A Toptica DL100/Pro tuneable diode laser was employed which has an advertised bandwidth of $<1.5 \times 10^{-6} \mathrm{~nm}$ [25]. The laser's emission was measured to have a FWHM of $7.5 \times 10^{-3} \mathrm{~nm}$ which was taken to be representative of the instrument broadening for this optical system.

The measured spectral line is a convolution of the $\mathrm{H}_{\alpha}$ emission function, $f_{\mathrm{H}}(\lambda)$, and the instrument function, $f_{A}(\lambda)$, such that the observed function, $f_{O}$, is given by:

$$
f_{O}(X)=\int_{-\infty}^{+\infty} f_{\mathrm{H}}(\lambda) f_{A}(X-\lambda) \cdot \mathrm{d} \lambda
$$

A simulated signal was constructed from the addition of seven individual fine structure signals, each broadened by a temperature, $T$, and convoluted with a Gaussian instrument function with a FWHM of $7.5 \times 10^{-3} \mathrm{~nm}$. A least squares fit was then used to solve for the value of $T$ that best matched the simulated spectra to the experimental data. Example fits for the Balmer alpha plasma emission at $460 \mathrm{~K}$ and $910 \mathrm{~K}$ are shown in figures 3(a) and (b) respectively. It should be noted that the observed higher temperatures are correlated with greater line intensity and therefore a greater signal to noise ratio. There is an indication of small 'wing' effects (discussed later) for the higher temperature plasma. The influence of the signal to 

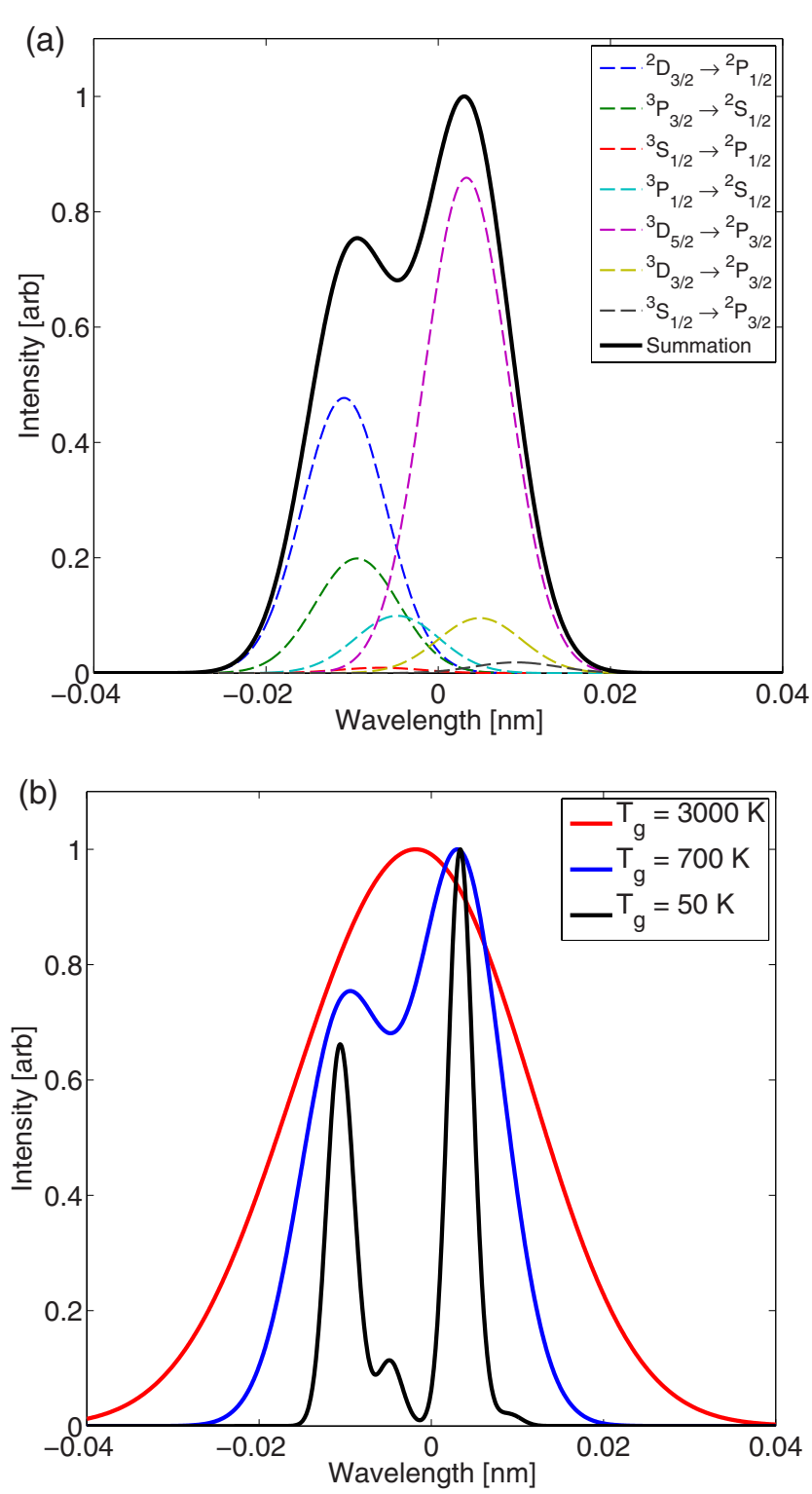

Figure 2. Theoretical $\mathrm{H}_{\alpha}$ profiles constructed from the individual fine structure transition profiles. (a) Individually Doppler broadened fine structure emissions (dashed) calculated at a temperature of $600 \mathrm{~K}$ as well as the resulting $\mathrm{H}_{\alpha}$ signal calculated by summing the individual gaussians. (b) Total emission profiles calculated for a number of temperatures.

noise ratio and the larger-than-ideal instrument broadening is reflected in the experimental uncertainty.

\subsection{Fulcher-band emission spectroscopy}

The determination of molecular gas temperatures from measurements of emission from ro-vibrational transitions is a well-established spectroscopic technique [26]. In the case of hydrogen, emission resulting from the Q-branch of Fulcher- $\alpha$ transitions is a popular method that has been applied in a number of plasma systems $[1,8,13,26-31]$. Fulcher- $\alpha$ emission is generated by molecular hydrogen transitioning from the $\mathrm{p}^{3} \Pi$ electronic state the to the $\mathrm{s}^{3} \Sigma$ state. The $\mathrm{Q}$ branch is the subset of Fulcher- $\alpha$ transitions that occurs without a change in total angular momentum of which the diagonal band also occur without change in vibrational quantum number. A schematic illustrating these transitions is shown in figure 4 and an example spectrum showing the emission resulting from these transitions and surrounding ro-vibrational transitions is presented in figure 5. Rotational temperatures were obtained by measuring the intensities of emission lines of individual rotational levels. By assuming a Boltzmann distribution amongst these rotational levels, the relative emission intensities were used to calculate the rotational temperature of the $\mathrm{p}^{4} \Pi$ state that was subsequently used to determine the translational temperature of the $s^{1} \Sigma$ ground state. However, there were a number of assumptions that must be made to correctly establish this relationship and care must be taken to ensure that the technique is correctly applied.

3.2.1. Technique assumptions. The assumptions required to relate the intensities of the Fulcher- $\alpha$ Q branch intensities to a ground-state translational temperature were outlined in detail by Gans et al [13]. They are discussed here in the context of the MAGPIE reactor.

(i) The ground state population must obey a Boltzmann distribution such that the rotational and translational temperatures are equal. If this is not the case, then a relationship between the rotational and translational temperatures must be defined.

(ii) Excited states must be populated solely by electron collisions from the ground state. To derive physical meaning from the emission of a particular molecular transition, knowledge of the population and depopulation mechanisms of the excited state must be known. This is straight-forward in the case of electron-impact excitation but becomes excessively complicated where multiple excitation mechanisms must be considered.

(iii) The adiabatic approximation must be valid for the transition being studied. Transfer of energy to the surrounding environment would otherwise need to be accounted for in the correlation between rotational temperatures of the ground and excited states. In general, the excited states of molecular hydrogen cannot be examined with adiabatic models with the notable exception being the Q-branch of the Fulcher series [32].

(iv) Rate coefficients must be independent of the rotational quantum number. Isolating a transition where there is no change in rotational quantum number is needed so that the equilibrium state identified in the $\mathrm{s}^{3} \Sigma_{\mathrm{g}}^{+}$state has a distribution of populations amongst rotational levels that is identical to that of the $\mathrm{p}^{3} \Pi_{\mathrm{u}}^{-}$level.

(v) The relaxation time of rotational levels must be longer than the lifetime of the excited state. This is required so that the population distribution amongst the rotational levels of the excited state cannot re-equilibrate before decay occurs. That is, they are still in a Boltzmann distribution defined by a temperature that can be related back to the translational temperature of the ground state. Furthermore, the excited state lifetime must be independent of the rotational quantum number so that decay of each level occurs at the same rate. 
(a)

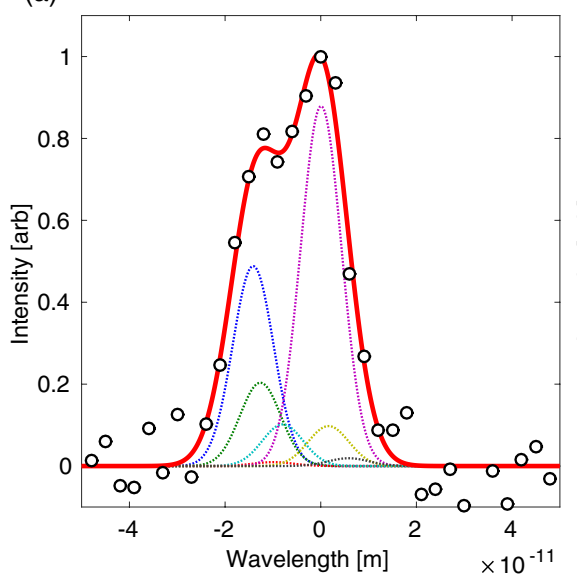

(b)

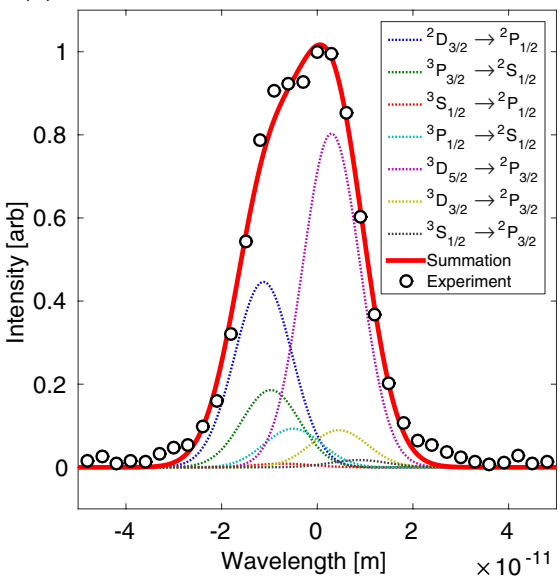

Figure 3. Example fine structure profile fits corresponding to atomic hydrogen gas temperatures of (a) $460 \mathrm{~K}$ and (b) $910 \mathrm{~K}$. Individual fine structure components (dashes) were summed to produce a predicted total emission (solid red line) that was fitted to experimental data (open circles).

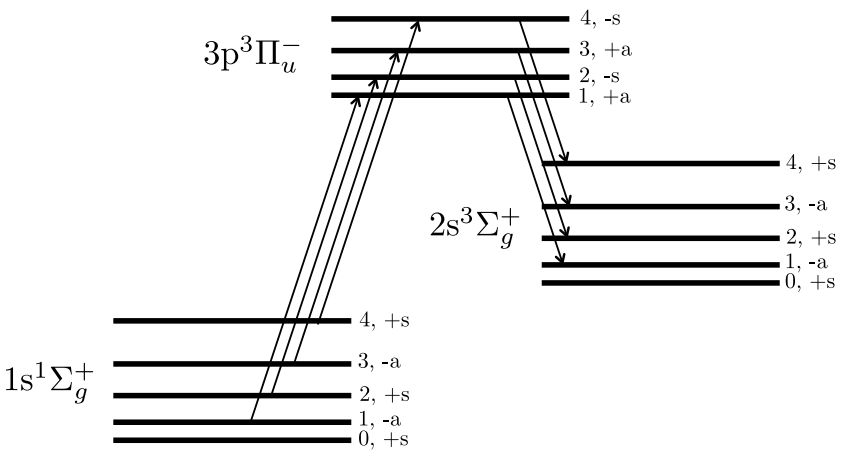

Figure 4. Term diagram illustrating the Q-branch of the Fulcher- $\alpha$ transition. This figure was produced using data from Gans et al [13]

Assumption (i) is generally considered valid in similar systems [13] and is reasonable when considering a ground-state population that will have sufficient time to reach an equilibrium. Assumption (ii) is valid in the Corona approximation, which assumes an equilibrium in which electron impact is responsible for ionisation and excitation, balanced by spontaneous emission and radiative recombination [33]. In general this is not the case in MAGPIE as the high electron density and low electron temperature dictate that there are a broad range of excitation and de-excitation mechanisms. However, a given electronic transition can be described by the Corona model if the population of the excited state is achieved mostly through electron-impact excitation and the decay is strictly radiative [27]. A criteria was developed to test this particular transition by Van der Mullen and described by de Graaf [27, 34]. It was found that for the purposes of the Fulcher- $\alpha$ band, the Corona approximation is considered valid for densities below $n_{\mathrm{e}}=6 \times 10^{19} \mathrm{~m}^{-3}$. The highest density measured in a hydrogen plasma in the MAGPIE reactor was $n_{\mathrm{e}}=1 \times 10^{19} \mathrm{~m}^{-3}$ and so the approximation was considered valid for typical MAGPIE conditions [16]. Assumptions (iii) and (v) are satisfied by the choice of Fulcher-Q transitions $[8,13,32]$. Assumption (iv) is not valid at low temperatures due to a broadening of the exited state's rotational distribution at low temperatures. However, this effect can be accounted for and is described in more detail below.
3.2.2. Technique application. Correlation between emission intensity and rotational temperature is achieved by applying the assumptions described above as well as some simplifications that resulted from the choice of the Fulcher- $\alpha$ Q band.

Consider first a molecule transitioning from an initial state $\left(e^{\prime}, v^{\prime}, J^{\prime}\right)$ to a final state $\left(e^{\prime \prime}, v^{\prime \prime}, J^{\prime \prime}\right)$ where $e$ denotes the electronic quantum number, $v$ the vibrational quantum number, and $J$, the rotational quantum number. The intensity of emission corresponding to this transition is given by [27]:

$$
I_{\left(e^{\prime \prime}, v^{\prime \prime}, J^{\prime \prime}\right)}^{\left(e^{\prime}, v^{\prime}, J^{\prime}\right)}=\frac{64 \pi \nu^{3}}{3 h} \frac{1}{2 J^{\prime}+1} N_{\left(e^{\prime}, v^{\prime}, J^{\prime}\right)} A_{\left(e^{\prime \prime}, v^{\prime}, J^{\prime \prime}\right)}^{\left(e^{\prime}, v^{\prime}, J^{\prime}\right)}
$$

where $\nu$ is the wavenumber, $h$ is planck's constant, $N_{\left(e^{\prime}, v^{\prime}, J^{\prime}\right)}$ is the population density of the initial state and $A$ is the Einstein transition probability of spontaneous emission. In the case of a Boltzmann distribution, the population amongst rotational states in the $\left(e^{\prime}, v^{\prime}\right)$ state will be governed by a rotational temperature $T_{\mathrm{rot}}\left(e^{\prime}, v^{\prime}\right)$ such that:

$$
N_{\left(e^{\prime}, v^{\prime}, J^{\prime}\right)}=c_{\left(e^{\prime}, v^{\prime}\right)} g_{a, s}\left(2 J^{\prime}+1\right) \times \exp \left(\frac{E_{\left(e^{\prime}, v^{\prime}, J^{\prime}\right)}}{k_{\mathrm{B}} T_{\mathrm{rot}\left(e^{\prime}, v^{\prime}\right)}}\right)
$$

where $c_{\left(e^{\prime}, v^{\prime}\right)}$ is a normalising constant, $g_{a, s}$ is the degeneracy of the $\left(e^{\prime}, v^{\prime}, J^{\prime}\right)$ state caused by the spin, $E_{\left(e^{\prime}, v^{\prime}, J^{\prime}\right)}$ is the energy, and $k_{\mathrm{B}}$ is Boltzmann's constant. The Einstein coefficient $A_{\left(e^{\prime \prime}, v^{\prime}, J^{\prime \prime}\right)}^{\left(e^{\prime}, y^{\prime}, J^{\prime}\right)}$ can be simplified by applying the Born-Oppenheimer approximation which states that a wavefunction can be separated into electronic, vibrational and rotational components [27]. In this case:

$$
A_{\left(e^{\prime \prime}, v^{\prime \prime}, J^{\prime \prime}\right)}^{\left(e^{\prime}, v^{\prime}\right)}=A_{e^{\prime}, e^{\prime \prime}} \times q_{v^{\prime}, v^{\prime \prime}} \times S_{J^{\prime}, J^{\prime \prime}}
$$

where $q_{v^{\prime}, v^{\prime \prime}}$ are Frank-Condon factors and $S_{J^{\prime}, J^{\prime \prime}}$ are HönlLondon factors. Substituting equations (5) and (6) into equation (4) gives:

$I_{\left(e^{\prime \prime}, v^{\prime \prime}, J^{\prime \prime}\right)}^{\left(e^{\prime}, J^{\prime} J^{\prime}\right)}=\frac{64 \pi \nu^{3}}{3 h} c_{\left(e^{\prime}, v^{\prime}\right)} g_{a, s} \exp \left(\frac{E_{\left(e^{\prime}, v^{\prime}, J^{\prime}\right)}}{k_{\mathrm{B}} T_{\mathrm{rot}\left(e^{\prime}, v^{\prime}\right)}}\right) \times A_{e^{\prime}, e^{\prime \prime}} q_{v^{\prime}, v^{\prime \prime}} S_{J^{\prime}, J^{\prime \prime}}$ 


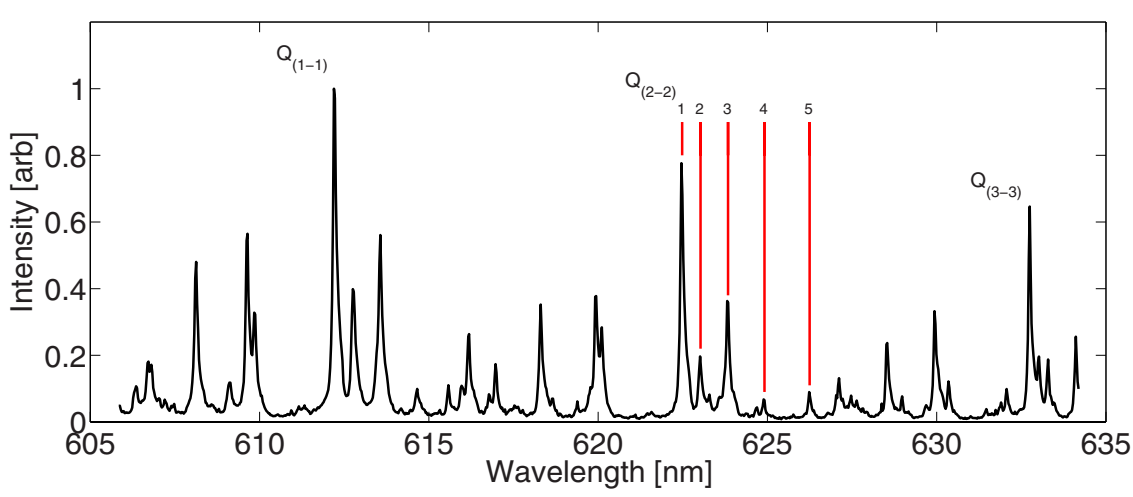

Figure 5. Emission profile measured $5 \mathrm{~mm}$ in front of a tungsten target in a $1000 \mathrm{~W} 10 \mathrm{mTorr}$ hydrogen plasma in the MAGPIE reactor. Bandheads for the 1-1, 2-2 and 3-3 Fulcher- $\alpha$ Q-branch emissions are labelled as are the first five rotational lines of the $v=2-2$ branch (red lines).

As $A_{e^{\prime}, e^{\prime \prime}}$ and $q_{v^{\prime}, v^{\prime \prime}}$ are constant with respect to the rotational quantum number in Fulcher Q diagonal transitions $\left(v^{\prime}=v^{\prime \prime}\right)$ and each transition undergoes an equivalent $\mathrm{p}^{3} \Pi \rightarrow \mathrm{s}^{3} \Sigma$ electronic transition, both terms vanish when describing the proportionality of the intensity with respect to the rotational temperature:

$$
I_{\left(e^{\prime \prime}, v^{\prime \prime} J^{\prime}\right)}^{\left(e^{\prime}, v^{\prime}\right)} \propto g_{a, s} \exp \left(\frac{E_{\left(e^{\prime}, v^{\prime}, J^{\prime}\right)}}{k_{\mathrm{B}} T_{\mathrm{rot}\left(e^{\prime}, v^{\prime}\right)}}\right) S_{J^{\prime}, J^{\prime \prime}}
$$

As described in detail by de Graaf, in the Fulcher- $\alpha$ series 'the orbital angular momentum and the angular momentum of nuclear rotation are coupled to the internuclear axis, but the electron spin is not' [27]. This scenario was described as case (b) in Hund's 1926 classification system [27, 35]. In this case, the Hönl-London factors for the Q-branch are simply:

$$
S_{J^{\prime}, J^{\prime \prime}}=\frac{2 J^{\prime}+1}{2}
$$

Substituting equation (9) and rearranging equation (8) gives:

$$
\ln \left(\frac{I_{\left(e^{\prime \prime}, v^{\prime \prime} J^{\prime \prime}\right)}^{\left(e^{\prime}\right)}}{g_{a, s}\left(2 J^{\prime}+1\right)}\right) \propto \frac{E_{\left(e^{\prime}, v^{\prime}, J^{\prime}\right)}}{k_{\mathrm{B}} T_{\mathrm{rot}\left(e^{\prime}, v^{\prime}\right)}}
$$

which demonstrates that there exists a relationship between the intensity of Fulcher- $\alpha$ rotational band emission and the rotational temperature that depends only on the rotational number, energy, and degeneracy of the initial state. This relationship was used to determine $T_{\text {rot }\left(e^{\prime}, v^{\prime}\right)}$ by plotting the LHS of the of equation (10) as a function of $E$. If the assumption of a Boltzmann distribution is accurate, a linear fit can be made from which the rotational temperature was taken directly from the slope of the fitted line. The degeneracy, $g_{a, c}$, was calculated as a function of the rotational quantum number such that: $g_{a, s}=1$ for even $J$ and $g_{a, s}=3$ for odd $J$. The energies $E_{\left(e^{\prime}, v^{\prime}, J^{\prime}\right)}$ were taken from the tables of Dieke [36]; the documented values for $\mathrm{s}^{1} \Sigma(v=0)$ were used as a consequence of assumption (v) above. For the rotational temperature of the excited state to be related to the translational temperature of the ground state, the transitions must occur fast enough that the rotational distribution cannot thermalise in its new state. The separation of energies amongst rotational bands in the $\mathrm{p}^{3} \Pi$ state will have been 'copied' from the ground-state and the use of the ground-state energies reflects this.

Two examples of the determination of an effective rotational temperature are shown in figure 6 for a MAGPIE plasma at a pressure of $10 \mathrm{mTorr}$ and at powers of (a) $1 \mathrm{~kW}$ and (b) $20 \mathrm{~kW}$. The larger magnitude slope in figure 6(a) compared to figure 6(b) is indicative of the smaller effective rotational temperature.

The rotational temperature of the ground state does not exactly equal the effective rotational temperature of the excited state due to broadening of the excited state's rotational distribution function compared to that of the ground state. Such a broadening is in contradiction with assumption (iv). The effect of this disparity is a 'hollowed' profile and an overestimation of the population density in higher rotational number states in the Boltzmann plot. As a result, the measured temperature is overestimated. The effect is more pronounced for lower temperatures; at $500 \mathrm{~K}$ in the ground-state plasma, this broadening results in an overestimation of the temperature by $40 \%$. This is taken into account by applying the simple correction suggested by de Graaf [27]. Measurements of the effective rotational temperature calculated using the linear fit method described here were multiplied by the correction factor calculated in de Graaf's work.

3.2.3. Diagnostic setup. A Princeton Instruments spectrograph and Princeton Instruments intensified charge coupled device (ICCD) camera were used for spectroscopic imaging of ro-vibrational bands. The basic setup for this system is shown schematically in figure 7. The Isoplane SCT-320 is a $320 \mathrm{~mm}$ focal length Schmidt-Czerny-Turner spectrograph with a $10 \mu \mathrm{m}$ to $12 \mathrm{~mm}$ entrance slit that was operated with a 1200 grooves $/ \mathrm{mm}$ diffraction grating with a $500 \mathrm{~nm}$ blazing. Connected to the Isoplane system was a PI-MAX $41024 \mathrm{i}$ ICCD camera. The PI-MAX 1024i features a $1024 \times 1024$ pixel array on a $13.08 \mathrm{~mm} \times 13.08 \mathrm{~mm}$ substrate cooled to $-20{ }^{\circ} \mathrm{C}$. The Isoplane/PI-MAX system is combined with a simple mirror and lens arrangement to image the plasma. Two 1-inch circular mirrors and a Nikon $f=200 \mathrm{~mm} \mathrm{1:4} \mathrm{camera}$ lens were used to capture an image of the region in front of a material sample placed inside the MAGPIE reactor. This image was centered at $r=0$ but needed to be rotated by $90^{\circ}$ 
(a)

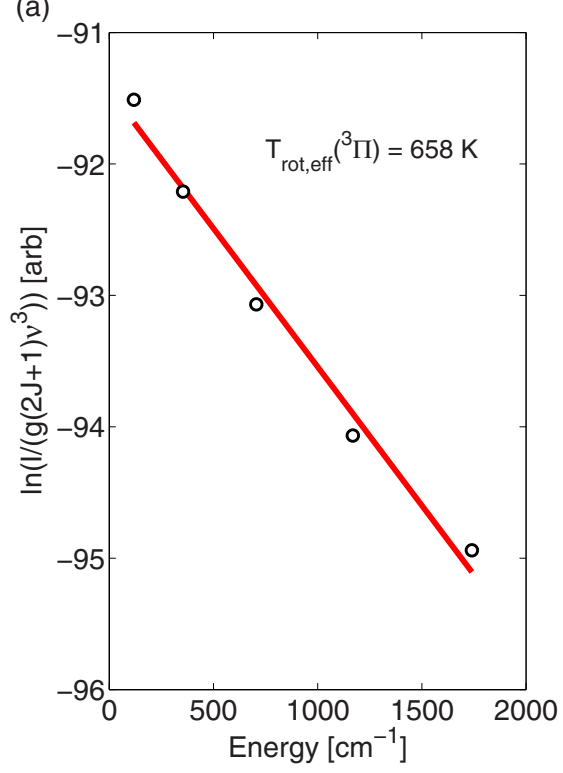

(b)

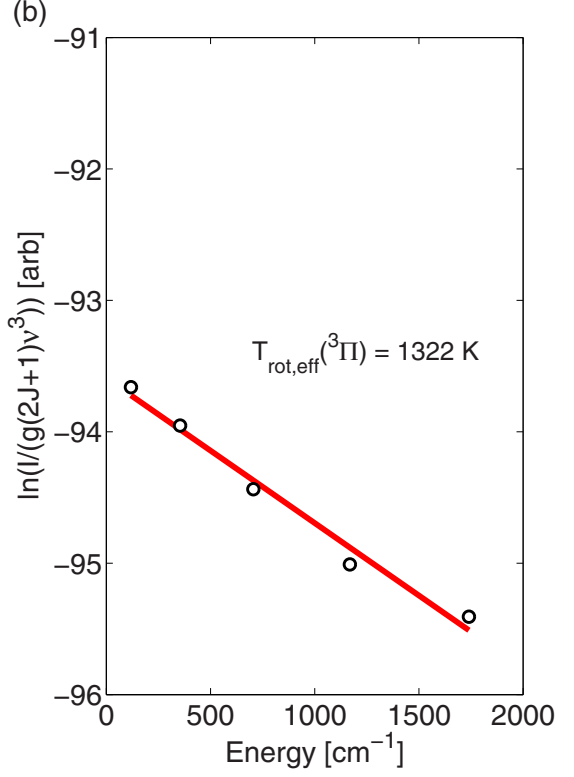

Figure 6. Boltzmann plots displaying the linear fits (red lines) used to determine effective rotational temperature of the ${ }^{3} \Pi$ state based on optical emission intensity measurements (circles) in a 10 mTorr plasma at powers of (a) $1 \mathrm{~kW}$, and (b) $20 \mathrm{~kW}$.

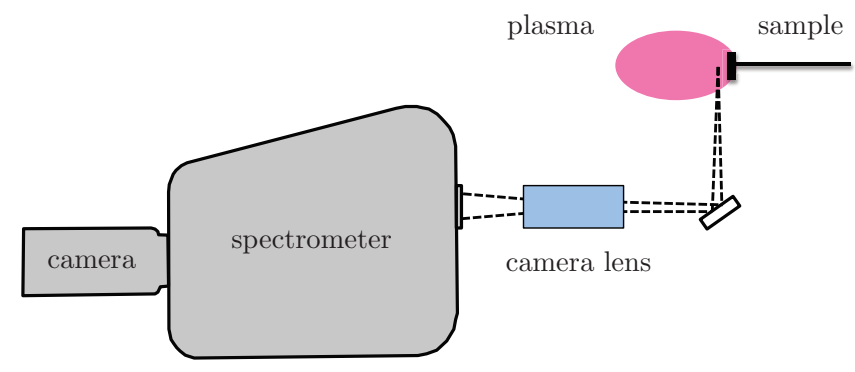

Figure 7. Schematic of the experimental setup used for spatially and temporally resolved spectroscopy measurements.

using a second mirror before being focused onto the spectrograph's slit. The spectrograph was set up in such a way that an image of the slit appeared on the CCD array. In this configuration, the spatial direction in front of the sample was resolved along one axis of the CCD image and the wavelength of light is resolved along the CCD's second axis. To focus the system, gridded paper was placed in the centre of the MAGPIE target chamber and the lens system adjusted to achieve a crisp image of the paper. This assured that the focus was aligned with the radial centre of both the target chamber and the material sample under investigation. A calibration of the spatial axis was achieved by calculating the number of pixels appearing between each $2 \mathrm{~mm}$ grid line. The spectrograph's slit was then narrowed to $10 \mu \mathrm{m}$ so that a well-characterised light source could be used to calibrate the system's spectral response. Princeton Instruments IntelliCal spectral sources were used for this purpose with a mercury lamp and a combined neon/ argon lamp used for wavelength calibrations. A broad spectrum LED source was used to calibrate the CCD and lens system's intensity response. An external trigger for the camera acquisition was supplied by a LabView control system that also controlled the plasma pulsing. Temporal delays were generated by the camera system allowing for timing accuracy down to 2 ns. Unless otherwise specified, acquired spectra were the sum of 50-200 on-CCD accumulations that were subsequently averaged 5 times.

Spatial resolution of the system depended largely on the choice of optics used to focus and magnify the plasma onto the spectrometer's entry slit. For the purpose of these measurements, the resolution was approximately $50 \mu \mathrm{m}$ per pixel. The largest source of uncertainty in molecular gas temperature measurements resulted from stochastic noise originating from the MAGPIE experiment. The magnitude of this noise was evaluated using a repeatability study whereby $T_{\mathrm{H}_{2}}$ was measured multiple times under the same conditions. A standard deviation of $22 \mathrm{~K}$ was found for measurements conducted in a typical $800 \mathrm{~W}, 10$ mTorr plasma. Some temperature results were obtained by averaging over a $5 \mathrm{~mm}$ region (approximately 100 measurements) in front of a sample surface. For these measurements an additional $22 \mathrm{~K}$ standard deviation was observed. Combining the standard error in the mean resulting from these standard deviation measurements resulted in an overall uncertainty of $\pm 20 \mathrm{~K}$ which is equivalent to approximately $5 \%$.

\section{Results}

\subsection{Low power operation}

Measurements of atomic and molecular hydrogen temperatures as a function of power and pressure in a low power regime were performed on the MAGPIE reactor. Applied powers for this regime were produced using the Alpha 77Dx $5 \mathrm{~kW}$ power supply in steady-state operation. Pressure scans were conducted at a power of $800 \mathrm{~W}$ and power scans were conducted at a pressure of 10 mTorr. A $1 \mathrm{~mm}$ thick, $32 \mathrm{~mm}$ diameter tungsten sample was loaded onto the sample holder and mounted in the MAGPIE target chamber. These experimental conditions were chosen to replicate those that were used in measurements of the atomic loss coefficient previously [17]. 


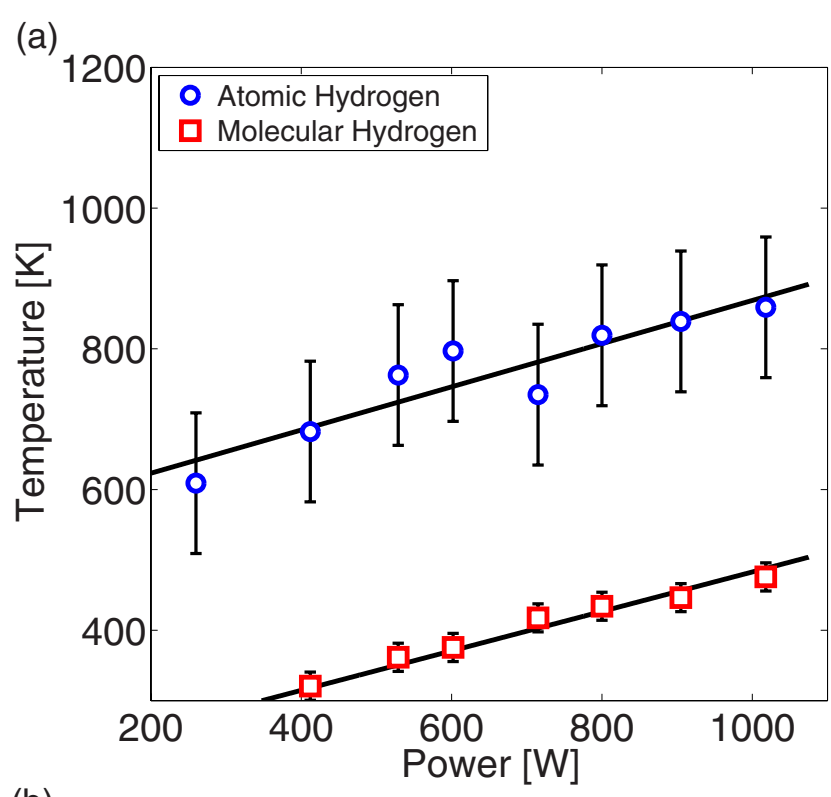

(b)

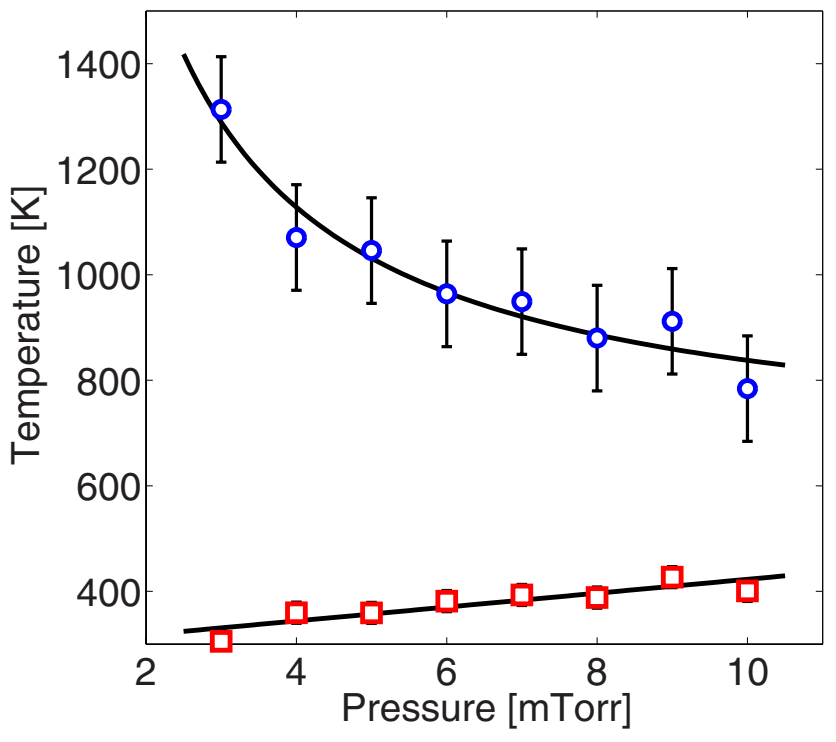

Figure 8. Atomic hydrogen temperature measurements (blue circles) and molecular hydrogen temperature measurements (red squares) as a function of (a) power and (b) pressure. Linear least squares fits are also shown (black lines) and described in the main text.

Doppler broadening and Fulcher- $\alpha$ measurements were made simultaneously to reduce the impact of stochastic variation in plasma conditions. For $\mathrm{H}_{\alpha}$ measurements, spectral emission from the plasma was collected using an optical fibre with an attached broad-acceptance lens and coupled into the spectrometer which was located remotely. The optical fibre was positioned so that the light admitted originated from the region between the tungsten sample and the source and so may be thought of as a 'bulk' measurement. Spatially resolved images of the Fulcher- $\alpha$ spectrum were acquired in front of the tungsten sample over a $100 \mathrm{~ms}$ period that encompassed 50 on-CCD measurements averaged five times. The total acquisition time for one measurement was approximately $30 \mathrm{~s}$. To reduce experimental uncertainty in measurements where spatial resolution wasn't required, the temperature was calculated by averaging over 100 pixels corresponding to a $5 \mathrm{~mm}$ region that starts $5 \mathrm{~mm}$ in front of the sample surface.

The results of these measurements are shown in figure $8 . T_{\mathrm{H}}$ was observed to range between $600 \mathrm{~K}$ and $850 \mathrm{~K}$ for powers between approximately 250 and $1000 \mathrm{~W}$. Between 400 and $1000 \mathrm{~W}, T_{\mathrm{H}_{2}}$ was observed to rise from 320 to $475 \mathrm{~K}$. The rise in temperature was linear in both cases and occurred at a rate of approximately $300 \mathrm{~K} \mathrm{~kW}^{-1}$. Extrapolating a linear line of best fit to a hypothetical zero-power case gave an atomic temperature of $560 \mathrm{~K}$ and a molecular temperature of around $200 \mathrm{~K}$. The non room-temperature $\mathrm{H}$ atom temperature can be explained by examining the production of atomic hydrogen. It is expected that atomic hydrogen would be almost entirely produced as a result of electron-impact dissociation ${ }^{1}$. In this reaction, $\mathrm{H}$ atoms are produced at an energy of $3.5 \mathrm{eV}$ [9]. This energy is rapidly dissipated through collisions, particularly with neutral molecules and its existence explains the relatively high temperatures observed at low powers. This energetic barrier doesn't exist for molecular hydrogen and so the 'zeropower' extrapolated value will necessarily be lower than that of hydrogen atoms that are 'born' at an energy far greater than the energy attributable to thermal motion. However, the predicted $200 \mathrm{~K}$ initial temperature for molecular hydrogen, being lower than room temperature, suggests the presence of a non-linearity at low powers that causes a convergence to room temperature, a low power coupling efficiency at low powers, or the break-down of the diagnostic technique in situations where the reduced emission intensity makes measurement difficult.

Measurements as a function of neutral pressure at an applied power of $800 \mathrm{~W}$ are shown in figure 8 (b). While the molecular temperature appears to rise as a function of increasing pressure, the atomic temperature decreases substantially over the 4-10 mTorr range. Least-squares fits to this data revealed that the molecular temperature's increase is approximately proportional to the pressure, rising from around $300 \mathrm{~K}$ at 3 mTorr to approximately $400 \mathrm{~K}$ at $10 \mathrm{mTorr}$. The atomic hydrogen temperature is inversely proportional to the pressure, decreasing from approximately $1300 \mathrm{~K}$ at 3 mTorr to $800 \mathrm{~K}$ at $10 \mathrm{mTorr}$. As was the case in the power dependency measurements, $T_{\mathrm{H}}$ is measured to be higher than $T_{\mathrm{H}_{2}}$ indicating that the two populations are not in thermal equilibrium.

Some comparisons can be made with relevant literature. Atomic hydrogen measurements by Aramaki et al in a $1.6 \mathrm{~cm}$ diameter, $30 \mathrm{~cm}$ long helicon plasma using the Doppler broadening of the $n=2$ to $n=3 \mathrm{H}_{\alpha}$ transition in a tuneable diode laser absorption spectroscopy (TDLAS) profile [6]. In their measurements, the atomic gas temperature increased as a function of power (1-3 kW at 50 mTorr) and decreased as a function of pressure (40-100 mTorr at $3 \mathrm{~kW})$. Trends were approximately linear in each case with temperatures measured in the range of 600-2300 K. While Aramaki's measurements were conducted at higher pressures, higher powers and in a smaller reactor, the trends agree broadly with the measurements presented here. Three spatial positions were examined by Aramaki whose results demonstrated that the atomic gas

${ }^{1}$ For more information, see [37]. 

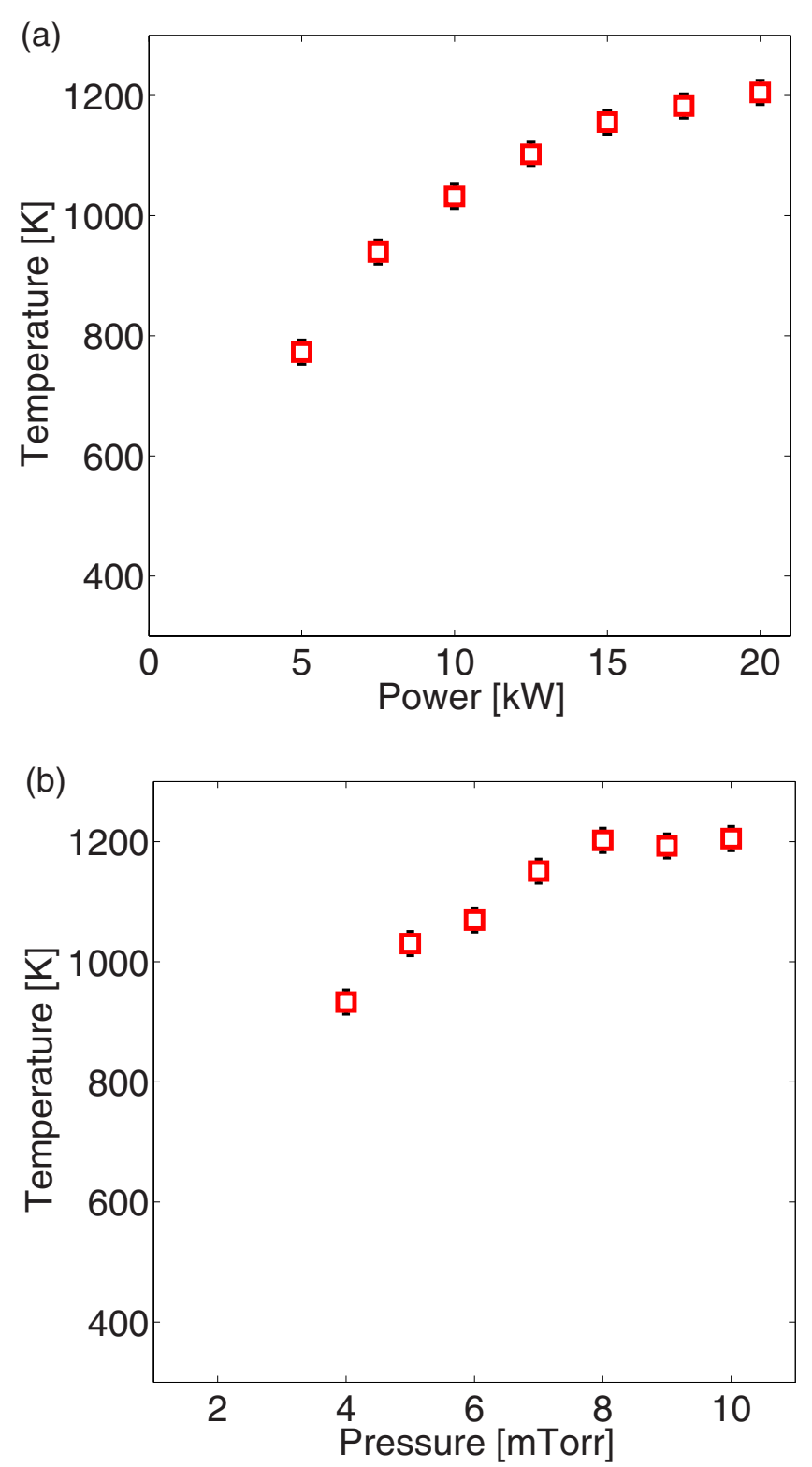

Figure 9. (a) Molecular gas temperatures as a function of power in a 10 mTorr plasma. (b) Molecular gas temperatures as a function of pressure in a $20 \mathrm{~kW}$ plasma.

temperature was higher in the central plasma column and decreased both in the diffusion chamber some distance from the antenna and outside of the plasma column. As such, it could be expected that a similar spatial dependence would be observed in the MAGPIE reactor, however, this effect is likely to be less pronounced at low pressures. Molecular hydrogen measurements were performed on the HELIOS IEC helicon reactor by Alderson and presented in his 2008 Masters dissertation [14]. The molecular gas temperature was found to be approximately $500 \mathrm{~K}$ at powers between 600 and $2100 \mathrm{~W}$ and at a pressure of approximately 70 mTorr. Galante et al measured atomic hydrogen temperatures using a two photon laser induced fluorescence (TALIF) in the CHEWIE reactor: a $60 \mathrm{~cm}$ long, $2.5 \mathrm{~cm}$ radius helicon reactor connected to a $20 \mathrm{~cm}$ long, $7.5 \mathrm{~cm}$ expansion chamber [15]. In this reactor, the atomic temperature was measured to be $0.08 \mathrm{eV}(\approx 930 \mathrm{~K})$ and it was reported that 'no changes in neutral temperatures were measured with varying source parameters' [38]. It is not known why the neutral temperatures were observed to be constant with respect to power and pressure in Galante and Alderson's measurements but found to vary considerably in Aramaki's measurements and in MAGPIE.

To the author's knowledge, the disparity between $T_{\mathrm{H}}$ and $T_{\mathrm{H}_{2}}$ has not been observed in radiofrequency discharges before, however the phenomenon has been observed in a number of other systems. Tomasini et al measured atomic temperatures that were $250 \mathrm{~K}$ above molecular temperatures in a microwave surfaguide experiment [11]. Pressures typically around 1 Torr and powers between 200 and $400 \mathrm{~W}$ were used with gas temperatures obtained from both Doppler broadening and analysis of molecular emission bands. To explain this difference, a Monte Carlo simulation was performed that calculated $\mathrm{H}$ atom velocity distribution functions. In addition to a low-velocity thermal component, a 'hot' population due to the $3.5 \mathrm{eV} \mathrm{H}$ atom fragments as the result of dissociation of $\mathrm{H}_{2}$ was observed at 50 mTorr. At higher pressures the smaller mean free path causes thermalisation of the velocity distribution function through collisions with molecules, other atoms and the walls. The degree to which this thermalisation occurs is dependent on the dissociation fraction, as molecules act to cool the more energetic $\mathrm{H}$ population. Following the results of Tomasini, it would be expected that in operating conditions that promote high dissociative fraction such as high power, low pressure or low recombination probability, the atomic hydrogen temperature would increase. Atomic temperatures measured to be in excess of molecular temperatures have also been observed in microwave-driven ICP reactors. Tatarova, Dias, and Ferreira [10] measured a considerable temperature difference in pure hydrogen as well as in $\mathrm{He}-\mathrm{H}_{2}$ and $\mathrm{Ar}-\mathrm{H}_{2}$ plasmas at a pressure of around 220 mTorr. The difference is attributed to both the hot $\mathrm{H}$ atoms from electron-impact dissociation and also from the $\mathrm{H}_{2}^{+}+\mathrm{H}_{2} \rightarrow \mathrm{H}_{3}^{+}+\mathrm{H}$ reaction, which produces $1.2 \mathrm{eV}$ atoms.

In other work, Felizardo et al [39] and Tatarova et al [40] demonstrated the presence of fast excited atoms with kinetic energies in the 4-9eV range. Spatially resolved measurements indicated that hot atoms are created near the wall by neutralization of positive hydrogen ions. Figure 3(b) may display an indication of broadened 'wing' formation, however it is not as pronounced as that observed in other works.

\subsection{High power operation}

One of the key advantages of the MAGPIE reactor is its ability to access high densities at low electron temperatures. At 20 $\mathrm{kW}$, and 3 mTorr, ion densities are on the order of $5 \times 10^{18} \mathrm{~m}^{-3}$ and the electron temperature is approximately $5 \mathrm{eV}$ [16]. High power helicon systems have not yet been comprehensively characterised; the measurement of neutral species temperatures presented here is the first work in this area.

While the increased complexity of pulsed operation and the limited exposure window precluded Doppler broadening measurements, the Fulcher- $\alpha$ technique was applied to 
measure the molecular gas temperature. Short pulse lengths necessitated a widening of the spectrograph's entry slit to $300 \mu \mathrm{m}$ so that a suitably high intensity of light incident on the CCD array was maintained. The slit-width was chosen to maximise light levels without losing the ability to resolve the Q-band transitions. As was the case in the low power measurements described previously, a $5 \mathrm{~mm}$ region was averaged. This region spans from $5 \mathrm{~mm}$ to $10 \mathrm{~mm}$ in front sample surface. For these measurements however, the process was conducted by adding the voltages across 100 CCD pixels (on-CCD accumulation) and not in post-processing to reduce acquisition time and to reduce experimental noise. The final $300 \mu$ s of the plasma pulse was acquired with 100 on-CCD accumulations that were subsequently averaged five times. All five rotational bands were measured during a single exposure to eliminate the influence of slow drifts in plasma conditions. This was somewhat mitigated by the low intensity of emission that required many exposures to gather enough light for adequate peak finding. In the case of long total acquisition time, measurements of each of the rotational emission lines concurrently leaves the ratio of intensities amongst these lines unchanged. The net effect is a linear averaging of the temperature over the acquisition period, not a perturbation in the resulting temperatures as could have occurred if each line was measured individually.

Molecular hydrogen measurements were obtained for a range of powers at a pressure of 10 mTorr and for a range of pressures at a power of $20 \mathrm{~kW}$. These measurements are shown in figure 9 . The molecular temperature was observed to rise as a function of power and pressure, as was the case in low power measurements. A rise in pressure from 4 to 8 mTorr produced an almost linear increase in gas temperature however the temperature appeared to be constant with respect to pressure between 8-10 mTorr. A maximum temperature of approximately $1200 \mathrm{~K}$ was observed at high powers and pressures, well in excess of the $\approx 500 \mathrm{~K}$ temperature observed at lower powers.

\subsubsection{Spatially resolved temperature in the near-target} region. The molecular temperature as a function of distance in front of a graphite target was measured at a number of powers and pressures and is presented in figure 10. The temperature was observed to rise as a function of pressure and power, as noted previously, and also as a function of distance in front of the target. In the region from about $2 \mathrm{~mm}$ in front of the sample surface to $10 \mathrm{~mm}$ in front of the sample surface, the increase in temperature was approximately linear with a higher temperature close to the source region and cooling observed in the vicinity of the target. Lines of best fit for this region are shown in figure 10. These linear fits demonstrate that the rate of change of temperature increases for increasing power but remains constant as a function of pressure. The lack of change in the slope's magnitude with respect to pressure, but significant change with respect to power may suggest the effect is linked to electron density and not electron temperature. The mean free path for $\mathrm{H}_{2}-\mathrm{H}_{2}$ collisions at 10 mTorr is approximately $1 \mathrm{~cm}$, suggesting that if electron heating is the
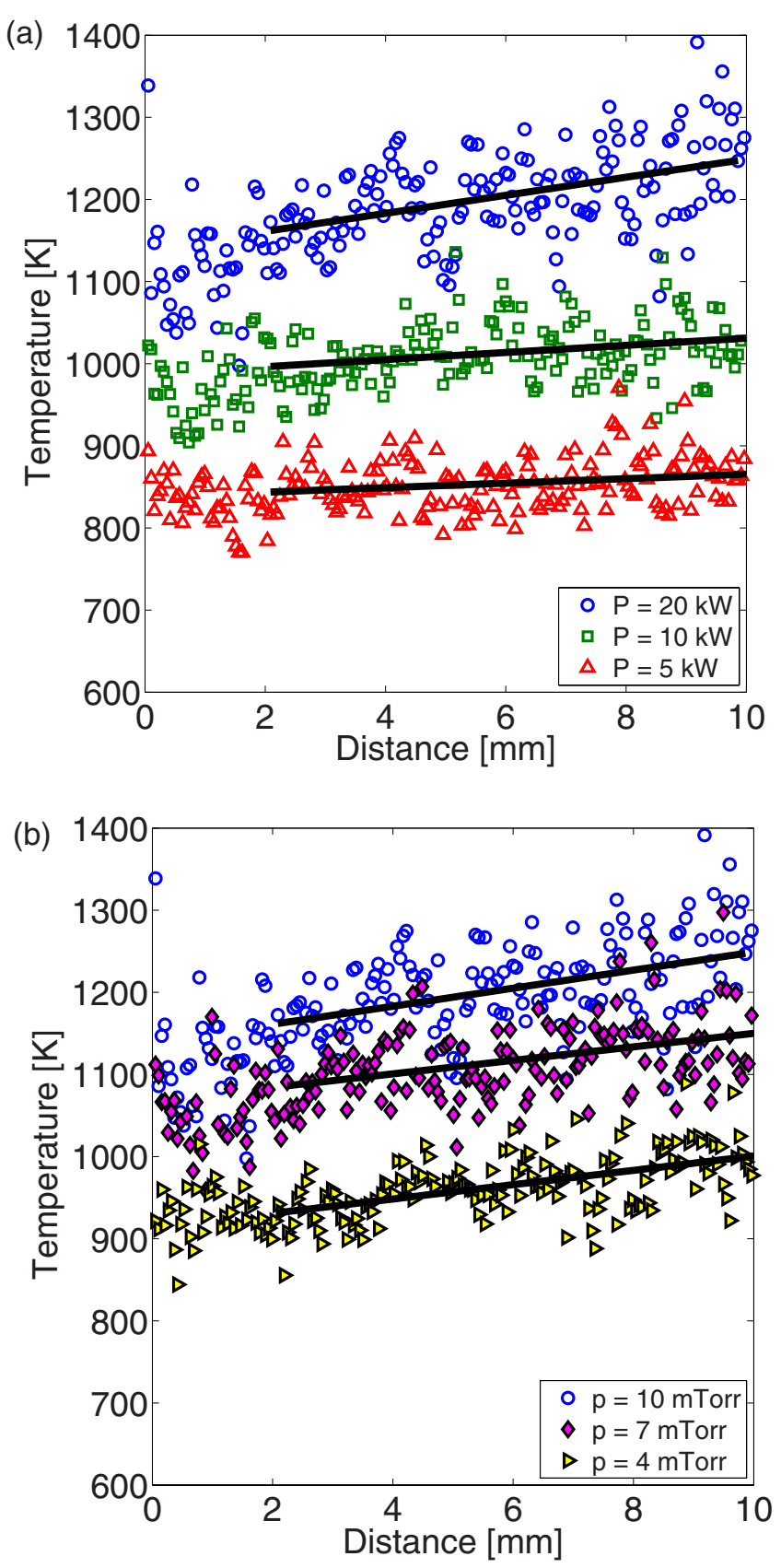

Figure 10. Molecular hydrogen temperature as a function of distance in front of a graphite sample in (a) 10 mTorr plasma at several powers and (b) a $20 \mathrm{kkW}$ plasma at several pressures. Linear fits made from $2 \mathrm{~mm}$ to $10 \mathrm{~mm}$ (black lines) are included to guide the eye.

cause of the decrease in neutral temperature, it is likely to be a localised effect. Atomic hydrogen measurements measured by Aramaki [6] at several positions downstream of a helicon antenna suggested decaying neutral temperature as a function of distance from the source region which also may be related to the phenomena measured in $\mathrm{H}_{2}$ temperatures here.

Close to the sample surface there is a region of non-linearity. It appears that the neutral temperature rises in the first few measurements $(50-100 \mu \mathrm{m})$ in front of the sample and then drops off before beginning the linear rise discussed above. The magnitude of this effect is smaller than the statistical temperature spread and so further investigation is required to 

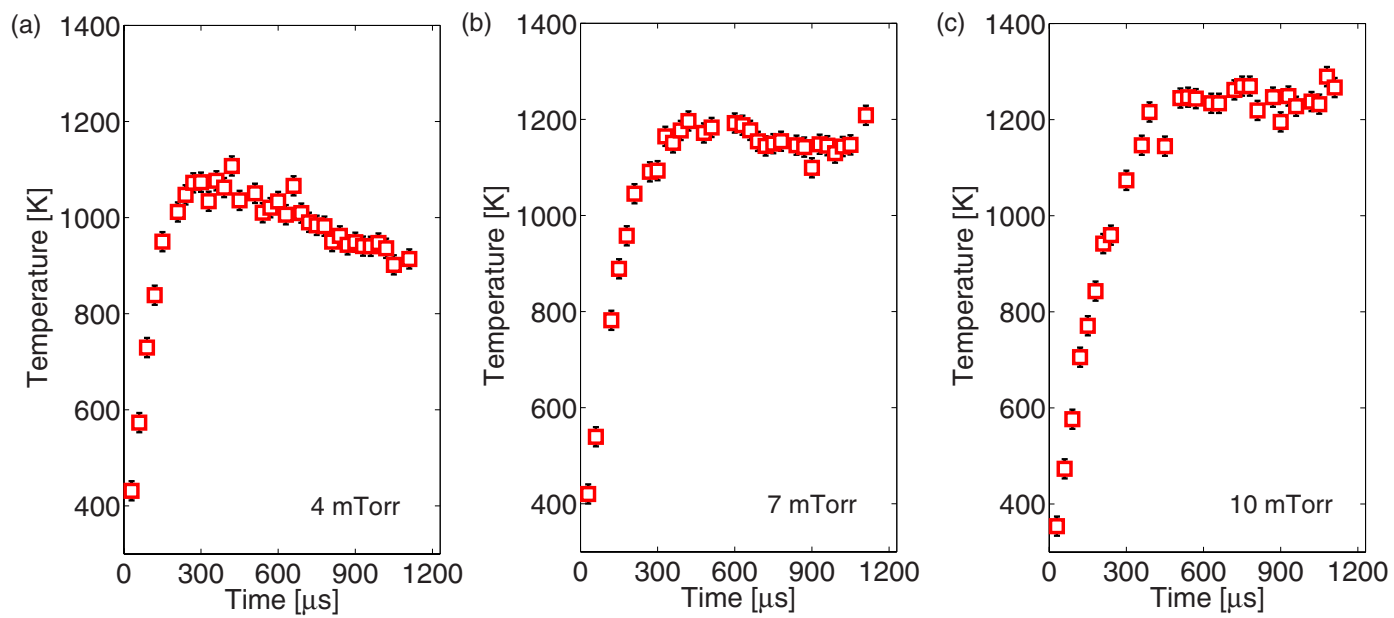

Figure 11. Molecular hydrogen gas temperature as a function of time in a $20 \mathrm{~kW}$, (a) 4 mTorr, (b) 7 mTorr, and (c) 10 mTorr discharge. The pulse length was approximately $1100 \mu$ s.

(a)

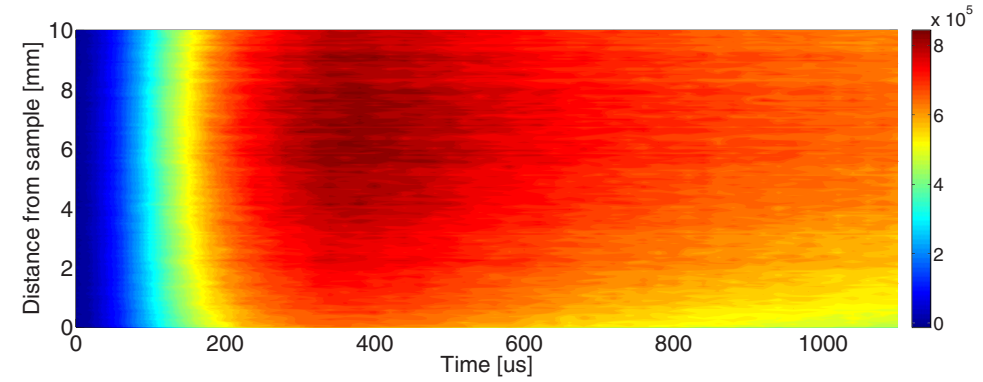

(b)

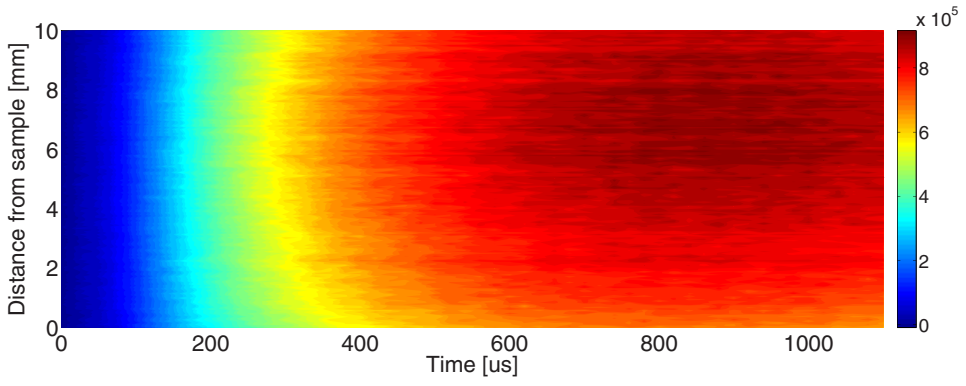

(c)
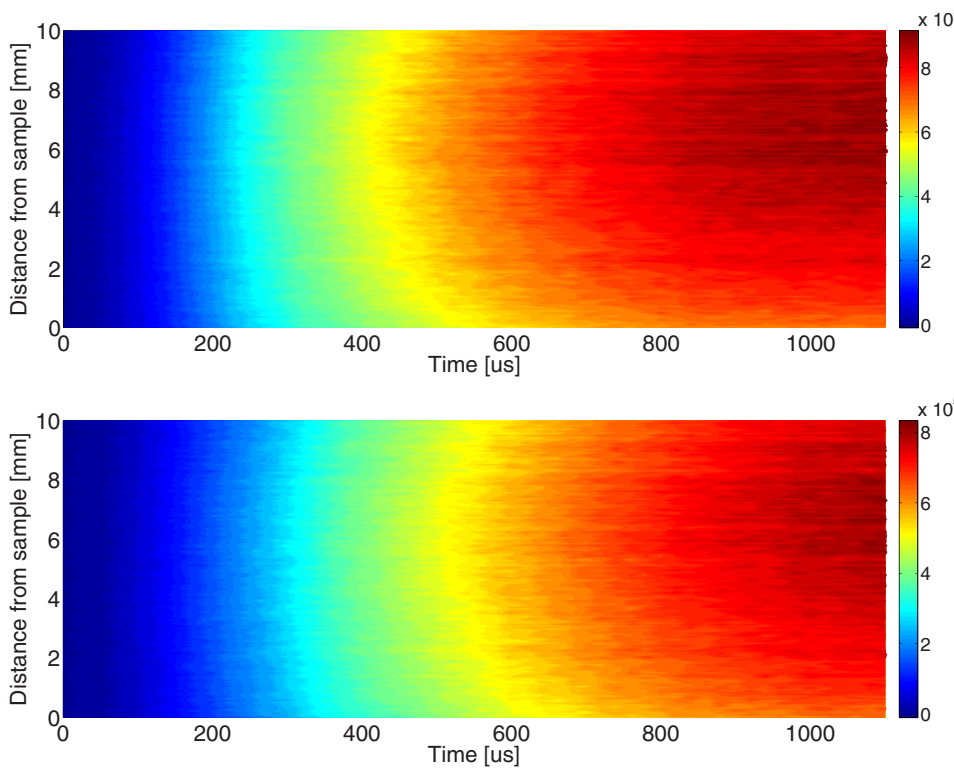

Figure 12. $\mathrm{H}_{\alpha}$ emission intensity as a function of time and distance in front of the sample surface. Measurements were made at $20 \mathrm{~kW}$ and at pressures of (a) 4 mTorr, (b) 6 mTorr, (c) 8 mTorr, and (d) 10 mTorr. 
confirm this profile. Given that the non-linearity occurs within a mean-free path for $\mathrm{H}_{2}-\mathrm{H}_{2}$ collisions and well outside the Debye length (approximately $1 \mathrm{~mm}$ assuming $T_{\mathrm{e}}=3 \mathrm{eV}$ and $n_{\mathrm{e}}=10^{18} \mathrm{~m}^{-2}$ ), it could be evidence of the sample's effect on the neutral dynamics in this region.

4.2.2. Plasma ignition profiles. To further investigate the temporal evolution, time-resolved temperatures for a $20 \mathrm{~kW}$ plasma at three pressures are shown in figure 11. These measurements occurred over a $30 \mu \mathrm{s}$ acquisition time accumulated 1000 times on the CCD array and then averaged four times with the entry slit set to a width of $30 \mu \mathrm{m}$. While it was observed that generally the temperature rises as a function of pressure, the ignition behaviour varies considerably. In the 10 mTorr case, the temperature rises to an equilibrium at around $600 \mu \mathrm{s}$ whereas in the $4 \mathrm{mTorr}$ case the temperature rises to a peak that occurs approximately $300 \mu \mathrm{s}$ into the plasma pulse and then decreases linearly until the plasma is extinguished at $1100 \mu \mathrm{s}$. The peak temperature is around 1100 $\mathrm{K}$ and then drops approximately $200 \mathrm{~K}$ before the plasma is extinguished. In the $7 \mathrm{mTorr}$ case, an intermediary behaviour was observed where the gas temperature peaks at around 400$600 \mu$ s and decreases by approximately $50 \mathrm{~K}$ before stabilising around $700 \mu$ s after plasma ignition.

To help explain this behaviour, $\mathrm{H}_{\alpha}$ emission under the same experimental conditions was measured using the same optical setup as used for the Fulcher- $\alpha$ measurements. The raw $\mathrm{H}_{\alpha}$ intensity at several pressures measured as a function of distance in front of the sample and time into a high powered pulse is shown in figure 12. A peak in emission intensity is observed around $7 \mathrm{~mm}$ from the sample surface that appears at earlier times for lower pressures. While not resolved for higher pressures, it appears that after an initial peak, the intensity drops off in a profile very similar to that observed in the low pressure gas temperature measurements.

The cause of the peak in $\mathrm{H}_{\alpha}$ emission and molecular hydrogen temperature at low pressures is not known. The appearance of similar profiles in both sets of measurements may be indicative of a peaked $n_{\mathrm{e}}$ or $T_{\mathrm{e}}$ profile, however further experiments are needed to delineate between the two parameters.

\section{Conclusion}

Measurements of the neutral temperatures in a low pressure RF discharge showed that the atomic and molecular neutral populations were not in thermal equilibrium. It was hypothesised that this is due to the energy imparted to dissociated atomic fragments. The temperature difference was constant as a function of applied power with both $T_{\mathrm{H}}$ and $T_{\mathrm{H}_{2}}$ rising a rate of about $300 \mathrm{~K} \mathrm{~kW}^{-1}$. For increasing pressure, the molecular hydrogen temperature was observed to rise linearly whereas the atomic hydrogen temperature dropped inversely with pressure.

Characterisation of the molecular hydrogen neutral temperature in a high power helicon system was performed. $T_{\mathrm{H}_{2}}$ was observed to rise as a function of applied power and pressure with a maximum temperature of around $1200 \mathrm{~K}$ measured in a $20 \mathrm{~kW}, 10 \mathrm{mTorr}$ plasma. Spatial temperature variations were observed in front of a graphite sample. Lower temperatures were observed near the sample surface which may be evidence of localised cooling. The rate of cooling as a function of distance was higher at high powers but was unaffected by changes in pressure. Evolution of the molecular hydrogen temperature was measured during a plasma pulse. At a pressure of 3 mTorr, the temperature rose to a peak of $1100 \mathrm{~K}$ over $300 \mu$ s before decaying to $900 \mathrm{~K}$ by the end of the $1.1 \mathrm{~ms}$ pulse. At the higher pressure of 10 mTorr, the temperature rises quickly before stabilising at $1200 \mathrm{~K}$ approximately $400 \mu$ s into the pulse. Similar profiles were observed in $\mathrm{H}_{\alpha}$ emission.

These measurements are important for our understanding of neutral dynamics in low pressure hydrogen plasmas. They also serve to demonstrate that there exists significant complexity in the behaviour of neutral gas temperatures that must be addressed in theoretical and modelling work.

\section{Acknowledgments}

CS Corr would like to acknowledge support from the Australian Research Council through a Future Fellowship (FT100100825). The authors acknowledge the support of the Australian Plasma Fusion Research Facility.

\section{References}

[1] Abdel-Rahman M, Gans T, Schulz von der Gathen V and Döbele H P 2005 Space and time resolved rotational state populations and gas temperatures in an inductively coupled hydrogen RF discharge Plasma Sources Sci. Technol. 1451

[2] Rousseau A, Teboul E and Sadeghi N 2004 Time-resolved gas temperature measurements by laser absorption in a pulsed microwave hydrogen discharge Plasma Sources Sci. Technol. 13166

[3] Shu W M, Kawasuso A and Yamanishi T 2009 Recent findings on blistering and deuterium retention in tungsten exposed to high-fluence deuterium plasma J. Nucl. Mater. 386 356-9

[4] Yamagiwa M, Kajita S, Ohno N, Takagi M, Yoshida N, Yoshihara R, Sakaguchi W and Kurishita H 2011 Helium bubble formation on tungsten in dependence of fabrication method J. Nucl. Mater. 417 499-503

[5] Hjartarson A T, Thorsteinsson E G and Gudmundsson J T 2010 Low pressure hydrogen discharges diluted with argon explored using a global model Plasma Sources Sci. Technol. 19065008

[6] Aramaki M, Okumura Y, Goto M, Muto S, Morita S and Sasaki K 2005 Measurements of gas temperature in highdensity helicon-wave $\mathrm{H}_{2}$ plasmas by diode laser absorption spectroscopy Japan. J. Appl. Phys. 446759

[7] Lee C and Lieberman M A 1995 Global model of Ar, $\mathrm{O}_{2}$, $\mathrm{Cl}_{2}$, and $\mathrm{Ar} / \mathrm{O}_{2}$ high-density plasma discharges $J$. Vac. Sci. Technol. 13 368-80

[8] Röpcke J and Ohl A 1994 Temperature distributions across the plasma layer of planar low pressure microwave plasmas comparative investigation by optical emission spectroscopy Contrib. Plasma Phys. 34 575-86

[9] Chabert P, Rousseau A, Gousset G and Leprince P 1998 On the influence of the gas velocity on dissociation degree and gas temperature in a flowing microwave hydrogen discharge J. Appl. Phys. 84 161-7

[10] Tatarova E, Dias F M, Puač N and Ferreira C M 2007 Hydrogen Balmer- $\alpha$ line broadening in a microwave plasma source Plasma Sources Sci. Technol. 16 S52 
[11] Tomasini L, Rousseau A, Gousset G and Leprince P 1996 Spectroscopic temperature measurements in a microwave discharge J. Phys. D: Appl. Phys. 291006

[12] Lamara T, Hugon R and Bougdira J 2006 Influence of gas temperature on the loss mechanisms of $\mathrm{H}$-atoms in a pulsed microwave discharge identified by time-resolved LIF measurements Plasma Sources Sci. Technol. 15526

[13] Gans T, Schulz-von der Gathen V and Döbele H F 2001 Time dependence of rotational state populations of excited hydrogen molecules in an RF excited plasma reactor Plasma Sources Sci. Technol. 1017

[14] Alderson E C 2008 Spectroscopic diagnosis of a dense hydrogen plasma source $P h D$ Thesis University of Wisconsin, Madison

[15] Galante M E, Magee R M and Scime E E 2014 Two photon absorption laser induced fluorescence measurements of neutral density in a helicon plasma Phys. Plasmas 21055704

[16] Blackwell B D, Caneses J F, Samuell C M, Wach J, Howard J and Corr C 2012 Design and characterization of the magnetized plasma interaction experiment (MAGPIE): a new source for plasma-material interaction studies Plasma Sources Sci. Technol. 21055033

[17] Samuell C M and Corr C S 2014 Ion flux dependence of atomic hydrogen loss probabilities on tungsten and carbon surfaces J. Nucl. Mater. 451 211-5

[18] Samuell C M, Blackwell B D, Howard J and Corr C S 2013 Plasma parameters and electron energy distribution functions in a magnetically focused plasma Phys. Plasmas 20034502

[19] Griem H R 1964 Plasma Spectroscopy 1 (New York: McGraw-Hill)

[20] Storm V P and Cappelli M A 1996 Stark broadening corrections to laser-induced fluorescence temperature measurements in a hydrogen arcjet plume Appl. Opt. 35 4913-8

[21] Lieberman M A and Lichtenberg A J 1994 Principles of Plasma Discharges and Materials Processing (New York: Wiley)

[22] Mills R L, Ray P C, Nansteel M, Chen Z, Mayo F M, He J and Dhandapani B 2003 Comparison of excessive balmer $\alpha$ line broadening of glow discharge and microwave hydrogen plasmas with certain catalysts IEEE Trans. Plasma Sci. 31 338-55

[23] Tatarova E, Dias F M, Ferreira C M and Puac N 2007 Spectroscopic determination of $\mathrm{H}, \mathrm{He}$ and $\mathrm{H}_{2}$ temperatures in a large-scale microwave plasma source J. Appl. Phys. 101063306

[24] NIST online database accessed August 2013 www.nist.gov/ $\mathrm{pml} /$ data/asd.cfm
[25] Accessed September 2013 www.toptica.com

[26] Surrey E and Crowley B 2003 Spectroscopic measurement of gas temperature in the neutralizer of the JET neutral beam injection system Plasma Physics and Controlled Fusion $\mathbf{4 5} 1209$

[27] de Graaf M J 1994 A new hydrogen particle source PhD Thesis Department of Physics, University of Technology, Eindhoven, Netherlands

[28] Rousseau A, Teboul E and Leprince P 1998 Time resolved temperature measurements in a $\mathrm{H}_{2}$ high power pulsed discharge J. Physique 8 Pr7 -287

[29] Gavare Z, Revalde G and Skudra A 2009 Plasma temperature determination of hydrogen containing high-frequency electrodeless lamps by intensity distribution measurements of hydrogen molecular band Int. J. Spectrosc. 2010

[30] Kado S, Yamasaki D, Xiao B, Iida Y, Okamoto A, Kajita S, Shikama T, Oishi T and Tanaka S 2006 On the anomalous characteristics in the $\mathrm{P}$ and $\mathrm{R}$ branches in a hydrogen Fulcher band J. Plasma Fusion Res. 7 54-8

[31] Majstorović G L, Šišović N M and Konjević N 2007 Rotational and vibrational temperatures of molecular hydrogen in a hollow cathode glow discharge Plasma Sources Sci. Technol. 16750

[32] Astashkevich S A, Käning M, Käning E, Kokina N V, Lavrov B P, Ohl A and Röpcke J 1996 Radiative characteristics of $3 \mathrm{p} \Sigma, \Pi ; 3 \mathrm{~d} \Pi^{-}, \Delta^{-}$states of $\mathrm{H}_{2}$ and determination of gas temperature of low pressure hydrogen containing plasmas J. Quant. Spectrosc. Radiat. Transfer 56 725-51

[33] Fantz U 2002 Atomic and molecular emission spectroscopy in low temperature plasmas containing hydrogen and deuterium. Habilitationschrift (Garching bei München: Mat Planck Institut für Plasma physik)

[34] Van der Mullen J 1990 Excitation equilibria in plasmas; a classification Phys. Rep. 191 109-220

[35] Hund F 1926 Zur deutung einiger erscheinungen in den molekelspektren Z. Phys. 36 657-74

[36] Dieke G H 1958 The molecular spectrum of hydrogen and its isotopes J. Mol. Spectrosc. 2 494-517

[37] Samuell C M 2014 Hydrogen plasmas and their interaction with fusion-relevant materials $P h D$ Thesis Australian National University

[38] Galante M E 2013 Two-photon absorption laser induced fluorescence measurements of neutral density in helicon plasma $P h D$ Thesis West Virginia University

[39] Felizardo E, Tatarova E, Henriques J, Dias F M, Ferreira C M and Gordiets B 2011 Energetic hydrogen atoms in wave driven discharges Appl. Phys. Lett. 99041503

[40] Tatarova E, Felizardo E, Dias F M, da Silva M L, Ferreira C M and Gordiets B 2009 Hot and super-hot atoms in a microwave plasma Appl. Phys. Lett. 95181503 